\title{
Effects of absorbents on growth performance, blood profiles and liver gene expression in broilers fed diets naturally contaminated with aflatoxin
}

\author{
J. B. Liu' ${ }^{1,2, *}$, H. L. Yan', S. C. Cao ${ }^{1}$, Y. D. Hu ${ }^{3}$, and H. F. Zhang ${ }^{2}$
}

\author{
* Corresponding Author: J. B. Liu \\ Tel: +86-0816-6089521, Fax: +86-0816-6089521 \\ E-mail: liuswust@163.com \\ 'School of Life Science and Engineering, Southwest \\ University of Science and Technology, Mianyang, \\ Sichuan 621010, China \\ ${ }^{2}$ State Key Laboratory of Animal Nutrition, Institute \\ of Animal Sciences, Chinese Academy of Agricultural \\ Sciences, Beijing 100193, China \\ ${ }^{3}$ Farm Animal Genetic Resources Exploration and \\ Innovation Key Laboratory of Sichuan Province, \\ Sichuan Agricultural University, Ya'an, Sichuan \\ 625014, China \\ ORCID \\ J. B. Liu \\ https://orcid.org/0000-0002-4302-6046 \\ H. L. Yan \\ https://orcid.org/0000-0002-2597-6902 \\ S. C. Cao \\ https://orcid.org/0000-0001-9797-241X \\ Y. D. Hu \\ https://orcid.org/0000-0003-3293-568X \\ H. F. Zhang \\ https://orcid.org/0000-0003-0177-8773
}

Submitted Nov 22, 2018; Revised Mar 31, 2019; Accepted Apr 20, 2019
Objective: The study was conducted to evaluate the effects of the absorbent (a mixture of activated carbon and hydrated sodium calcium aluminosilicate) on growth performance, blood profiles and hepatic genes expression in broilers fed diets naturally contaminated with aflatoxin.

Methods: A total of 1,200 one-day-old male chicks were randomly assigned to 6 treatments with 10 replicate cages per treatment. The dietary treatments were as follows: i) control (basal diets); ii) $50 \%$ contaminated corn; iii) $100 \%$ contaminated corn; iv) control $+1 \%$ adsorbent; v) $50 \%$ contaminated corn $+1 \%$ absorbent; vi) $100 \%$ contaminated corn $+1 \%$ absorbent.

Results: During d 1 to 21 , feeding contaminated diets reduced $(\mathrm{p}<0.05)$ body weight $(\mathrm{BW})$, average daily gain $(\mathrm{ADG})$, and average daily feed intake (ADFI), but increased $(\mathrm{p}<0.05)$ feed-to-gain ratio $(\mathrm{F} / \mathrm{G})$. The absorbent supplementation increased $(\mathrm{p}<0.05) \mathrm{BW}, \mathrm{ADG}$, and ADFI. There were interactions ( $\mathrm{p}<0.05)$ in BW, ADG, and ADFI between contaminated corn and absorbent. Overall, birds fed $100 \%$ contaminated diets had lower $(\mathrm{p}<0.05)$ final BW and ADG, but higher $(\mathrm{p}<0.05) \mathrm{F} / \mathrm{G}$ compared to those fed control diets. The absorbent addition increased $(\mathrm{p}<0.05)$ serum albumin concentration on $\mathrm{d} 14$ and 28 and total protein $(\mathrm{TP})$ level on $\mathrm{d} 28$, decreased $(\mathrm{p}<0.05)$ alanine transaminase activity on $\mathrm{d} 14$ and activities of aspartate aminotransferase and alkaline phosphatase on $\mathrm{d} 28$. Feeding contaminated diets reduced $(\mathrm{p}<0.05)$ hepatic TP content on $\mathrm{d} 28$ and 42 . The contaminated diets upregulated $(\mathrm{p}<0.05)$ expression of interleukin-6, catalase (CAT), and superoxide dismutase (SOD), but downregulated $(\mathrm{p}<0.05)$ glutathione $\mathrm{S}$-transferase (GST) expression in liver. The absorbent supplementation increased $(\mathrm{p}<0.05)$ interleukin-1 $\beta$, CAT, SOD, cytochrome P450 1A1 and GST expression in liver. There were interactions $(\mathrm{p}<0.05)$ in the expression of hepatic CAT, SOD, and GST between contaminated corn and absorbent.

Conclusion: The results suggest that the naturally aflatoxin-contaminated corn depressed growth performance, while the adsorbent could partially attenuate the adverse effects of aflatoxin on growth performance, blood profiles and hepatic genes expression in broilers.

Keywords: Aflatoxin; Adsorbent; Broilers; Gene Expression; Growth Performance; Liver

\section{INTRODUCTION}

Aflatoxins (AFB), produced by Aspergillus flavus and Aspergillus parasiticus, are the most commonly occurring mycotoxins in poultry feed [1]. Aflatoxin $\mathrm{B}_{1}\left(\mathrm{AFB}_{1}\right)$, aflatoxin $\mathrm{B}_{2}\left(\mathrm{AFB}_{2}\right)$, aflatoxin $\mathrm{G}_{1}\left(\mathrm{AFG}_{1}\right)$, and aflatoxin $\mathrm{G}_{2}\left(\mathrm{AFG}_{2}\right)$ are the major forms of $\mathrm{AFB}$, of which $\mathrm{AFB}_{1}$ is considered the most toxic [2]. The occurrence of AFB poses a great threat to broiler health because of its adverse effects on growth performance, immunity, intestinal morphology, blood profiles and hepatic functions [3-6]. A meta-analysis study indicated that AFB-contaminated diets caused $11 \%$ reduction in feed intake and daily weight gain in broilers, and 
the feed conversion ratio was also decreased by $6 \%$ [7].

It is therefore important to minimize the AFB exposure to prevent its detrimental effects. Recently, strategies, such as adsorption method, biodegradation and chemical treatment, have been proposed to alleviate the toxic effects of mycotoxins on livestock [3,8-9]. Among them, supplementation of adsorbent is the most suitable approach to detoxify mycotoxins-contaminated feed, because it can bind and immobilize mycotoxins to reduce toxic residues in feed and effectively prevent health risks in animals [10]. Hydrated sodium calcium aluminosilicate (HSCAS), as one of the adsorbents, has been proved to be effective in adsorbing AFB [11]. Activated carbon, another adsorbent, could improve body weight (BW) gain and feed conversion ratio of chickens fed AFB-contaminated diets [12]. However, the efficacy of a combination of the above two adsorbents has not been examined in broilers fed diets with AFB.

It is well documented that naturally contaminated diets were more toxic than diets containing purified mycotoxins. Nevertheless, few researches were conducted on the effect of naturally AFB-contaminated diets containing low levels of $\mathrm{AFB}_{1}$ and $\mathrm{AFB}_{2}$ on broilers $[13,14]$. The liver is a principal target organ of $\mathrm{AFB}$ and is also responsible for detoxification of AFB [15]. Previous study in broilers demonstrated that the liver would present pathological lesions and hepatic functions damage when the content of $\mathrm{AFB}_{1}$ was high (1 to $5 \mathrm{mg} / \mathrm{kg}$ ) [4]. Notwithstanding, few literatures have examined the effect of corn naturally contaminated with low levels of $\mathrm{AFB}_{1}$ and $\mathrm{AFB}_{2}$ on the health and liver gene expression of broilers $[5,16]$. Furthermore, the effect of combined adsorbent on the response to naturally AFB-contaminated diets for broilers, with expected alleviation of toxicosis, has not yet been investigated. Therefore, the objective of this study was to determine the effect of the absorbent (a combination of activated carbon and HSCAS) on growth performance, blood profiles, liver weight and total protein level (TP) as well as liver gene expression in broilers fed diets with 50\% and 100\% naturally AFB-contaminated corn.

\section{MATERIALS AND METHODS}

\section{Experimental design, broilers, and diets}

The Animal Welfare Committee of China Agricultural University approved the animal care protocol used for this experiment. A total of 1200 one-day-old male broiler chicks (Cobb) with an average initial BW of $45.1 \pm 0.3 \mathrm{~g}$ were randomly assigned to 1 of 6 treatments in a $3 \times 2$ factorial arrangement with 3 levels of AFB-contaminated corn $(0 \%, 50 \%$, and $100 \%)$ and 2 absorbent levels ( $0 \%$ and $1 \%$ ) for a $42-\mathrm{d}$ study period. There were 10 replicates (cages) per treatment and 20 birds per replicate. The experiment lasted for 6 weeks, consisting of a starter phase from d 1 to 21 and a grower phase from $\mathrm{d} 22$ to 42 . All diets (Table 1) were formulated to meet or exceed the NRC requirements for broilers [17]. All birds were housed in an environmentally controlled facility. Diets were fed in pellet form and feed and water were provided ad libitum throughout the experiment. The absorbent, which consisted of equal amount of activated carbon and HSCAS, was added at the expense of corn.

All the feed samples were stored at $-20^{\circ} \mathrm{C}$ until further analyses. Concentrations of dry matter (DM) and nitrogen $(\mathrm{N})$ in the feed were analyzed in accordance with AOAC procedures [18]. The DM of the feed was determined after drying for $24 \mathrm{~h}$ at $103^{\circ} \mathrm{C}$. The $\mathrm{N}$ content was determined by using a Kjeltec 2300 Analyzer (Foss Tecator AB, Hoeganaes, Sweden). The ash content was determined after the ignition of a weighed sample in a muffle furnace (Nabertherm, Bremen, Germany) at $550^{\circ} \mathrm{C}$ for $6 \mathrm{~h}$. The ash was then digested in aqua regia $\left(\mathrm{HCl} / \mathrm{HNO}_{3}\right.$ mixture), and the solution was used for calcium $(\mathrm{Ca})$ and phosphorus $(\mathrm{P})$ determinations. Ca concentration was determined using an atomic absorption spectrophotometer (Varian'50, Varian, Palo Alto, CA, USA), and the concentration of $\mathrm{P}$ was determined spectrophotometrically (NanoDrop 2000c, Thermo Scientific, Delaware, MA, USA)

Table 1. Diet composition (as-fed basis)

\begin{tabular}{|c|c|c|}
\hline Items & Starter (d 1 to 21 ) & Grower (d 22 to 42 ) \\
\hline \multicolumn{3}{|l|}{ Ingredients (\%) } \\
\hline Corn & 56.16 & 59.95 \\
\hline Soybean meal (CP 44\%) & 31.50 & 25.45 \\
\hline Corn gluten meal (CP 60\%) & 4.65 & 5.02 \\
\hline Tallow & 3.50 & 5.50 \\
\hline Limestone & 1.00 & 1.00 \\
\hline Dicalcium phosphate & 2.08 & 1.93 \\
\hline $\mathrm{NaCl}$ & 0.40 & 0.40 \\
\hline L-Lys·HCl (24\%) & 0.20 & 0.20 \\
\hline DL-methionine (99\%) & 0.20 & 0.20 \\
\hline L-threonine $(98.5 \%)$ & 0.15 & 0.15 \\
\hline Vitamin premix ${ }^{1)}$ & 0.10 & 0.10 \\
\hline Trace mineral premix ${ }^{1)}$ & 0.10 & 0.10 \\
\hline \multicolumn{3}{|l|}{ Analytical composition } \\
\hline ME $(\mathrm{kcal} / \mathrm{kg})^{2)}$ & 3,050 & 3,200 \\
\hline Crude protein (\%) & 22.03 & 19.98 \\
\hline Lysine $(\%)$ & 1.17 & 1.05 \\
\hline Methionine (\%) & 0.59 & 0.55 \\
\hline Methionine+cystine (\%) & 0.86 & 0.72 \\
\hline Threonine (\%) & 0.78 & 0.74 \\
\hline $\mathrm{Ca}(\%)$ & 0.93 & 0.88 \\
\hline Total P (\%) & 0.72 & 0.67 \\
\hline \multicolumn{3}{|c|}{ 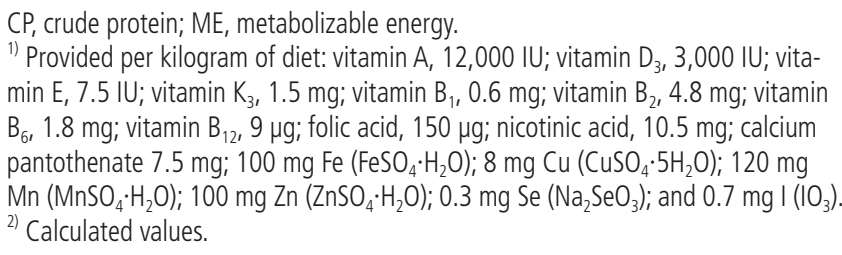 } \\
\hline
\end{tabular}


as previously described [19]. Amino acid contents were determined, following acid hydrolysis with $6 \mathrm{~N} \mathrm{HCl}$ at $110^{\circ} \mathrm{C}$ for $24 \mathrm{~h}$, using an amino acid analyzer (Biochrom 20, Pharmacia Biotech, Cambridge, England) [20]. Before acid hydrolysis, methionine was oxidized with formic acid.

\section{Analysis of mycotoxins}

The mycotoxins concentrations in the corn and diet were analyzed by HPLC (Agilent1100, Agilent Technologies, Santa Clara, CA, USA) according to AOAC method [18]. The detection limits of mycotoxins were $2 \mu \mathrm{g} / \mathrm{kg}$ for $\mathrm{AFB}_{1}, 0.8 \mu \mathrm{g} / \mathrm{kg}$ for $\mathrm{AFB}_{2}, 2.5 \mu \mathrm{g} / \mathrm{kg}$ for $\mathrm{AFG}_{1}, 1.5 \mu \mathrm{g} / \mathrm{kg}$ for $\mathrm{AFG}_{2}, 300 \mu \mathrm{g} / \mathrm{kg}$ for deoxynivalenol (DON), $100 \mu \mathrm{g} / \mathrm{kg}$ for zearalenone (ZEA), $30 \mu \mathrm{g} / \mathrm{kg}$ for T-2 toxin, $100 \mu \mathrm{g} / \mathrm{kg}$ for ochratoxin A (OTA), and $200 \mu \mathrm{g} / \mathrm{kg}$ for fumonisin $\mathrm{B}_{1}\left(\mathrm{FB}_{1}\right)$.

\section{Sample collection and measurements}

The birds were weighed, and feed intake was recorded on $\mathrm{d}$ 1,21 , and 42 , and average daily gain (ADG), average daily feed intake (ADFI), and feed-to-gain ratio (F/G) were calculated [21]. Mortality was recorded as it occurred. Both ADFI and $\mathrm{F} / \mathrm{G}$ were corrected for mortality.

On d 14, 28, and 42, 2 birds from each replicate were randomly selected from each cage and blood samples were collected from the jugular vein into a sterile syringe and stored at $-4^{\circ} \mathrm{C}$. Blood samples were then centrifuged at $3,000 \times \mathrm{g}$ for $15 \mathrm{~min}$ and serum was separated. The levels of TP, albumin (ALB), alanine transaminase (ALT), and aspartate aminotransferase (AST), alkaline phosphatase (AKP), and $\gamma$-glutamyl transferase $(\gamma-G T)$ in the serum were measured using the colorimetric method (Jiancheng Biotechnology Institute, Nanjing, China) following the kit instructions.

After blood collection, the same birds were weighed individually, and then sacrificed by cervical dislocation and exsanguinated. Livers were removed by trained personnel and weighed, and the relative liver weight was calculated as a percentage of BW. Liver samples were collected and then stored at $-20^{\circ} \mathrm{C}$ until required. All liver samples were determined for TP by the colorimetric method according to the manufacturer's instructions (Nanjing Jiancheng Bioengineering Institute, Nanjing, China).

The expression of interleukin-6 (IL-6), IL- $1 \beta$, interferon- $\gamma$ (IFN- $\gamma$ ), catalase (CAT), superoxide dismutase (SOD), glutathione peroxidase (GSH-Px), cytochrome P450 1A1 (CYP1A1), epoxide hydrolase (EH) and glutathione S-transferase (GST) in liver on $\mathrm{d} 42$ were analyzed using quantitative real-time polymerase chain reaction (PCR) (MyiQ real-time PCR detection system, Bio-Rad, Hercules, CA, USA) with SYBR mix (Bio-Rad, USA). Briefly, total RNA from hepatocytes (16 samples per treatment) was extracted using TRIzol (Invitrogen, Carlsbad, CA, USA). Extracted RNA was quantified at an absorbance of $260 \mathrm{~nm}$ (ND-1000, Nanodrop Technologies Inc., Wilmington, DE, USA) [22]. The reverse transcription was carried out using the M-MLV reverse transcriptase (Promega, Madison, WI, USA). The standard curve was established using pooled samples, and water served as a negative control. All reactions were analyzed in duplicate and the formation of a single PCR product was confirmed by melting curves [23]. The primer sequences were designed using Primer3 software (http://primer3.wi.mit.edu; Table 2). The mRNA expression was determined from the threshold cycle for respective genes, and the expression level was calculated using the $\Delta \Delta \mathrm{Ct}$ method normalized using glyceraldehyde phosphate dehydrogenase $(G A P D H)$ expression [24].

\section{Statistical analysis}

A two-way analysis of variance of the data using the general linear model procedure of SAS statistical software (SAS Inst. Inc., Cary, NC, USA) was performed according to a $3 \times 2$ factorial arrangement of treatments, including contaminated corn level and absorbent level as the main effects and respective interaction. Pen was considered as the experimental unit for all measurements. Differences among treatment means were separated using the least significant difference test at $\mathrm{p}<$

Table 2. Primer sequences $\left(5^{\prime} \rightarrow 3^{\prime}\right)$ used in quantitative real-time polymerase chain reaction

\begin{tabular}{lll}
\hline Genes & \multicolumn{1}{c}{ Forward primer } & \multicolumn{1}{c}{ Reverse primer } \\
\hline GAPDH & TCCTAGGATACACAGAGGACCA & CGGTTGCTATATCCAAACTCA \\
SOD & AGGGGGTCATCCACTTCC & CATTTGTGTTGCTCCAA \\
$E H$ & AAAGGGACAGAAGCCTGACA & CCTCCAGTGGCTCAGTGAAT \\
$I L-6$ & GAATGTTTAGTTCGGGCACA & TTCCTAGAAGGAATGAGAATGC \\
$I L-1 \beta$ & GCATCAAGGGCTACAAGCTC & CAGGCGGTAGAAGATGAAGC \\
$I F N-\gamma$ & CAAGTAATTGGATGTAGC & GCGTTGGATTTCAAGCC \\
CAT & GGGGAGCTGTTTACTGCAAG & TTTCCATTGGCTATGGCATT \\
GSH-PX & TTGTAAACATCAGGGGCAAA & TGGGCCAAGATCTTTCTGTAA \\
GST & GCCTGATGCACTTGCAAAA & AAAATTGCCATCAGTCTTGGT \\
CYPIA1 & CACTTTCTGCCTGCTCCTG & GGTCCTTCCTCAGCTCCAG
\end{tabular}

GAPDH, glyceraldehyde phosphate dehydrogenase; SOD, superoxide dismutase; $E H$, epoxide hydrolase; IL, interleukin; IFN- $\gamma$, interferon- $\gamma$; CAT, catalase; GSH-PX, glutathione peroxidase; GST, glutathione S-transferase; CYP1A1, cytochrome P450 1A1. 
Table 3. Concentrations of $\mathrm{AFB}_{1}$ and $\mathrm{AFB}_{2}$ in corn and diets

\begin{tabular}{|c|c|c|c|c|}
\hline \multirow{2}{*}{ Items $(\mu \mathrm{g} / \mathrm{kg})$} & \multicolumn{2}{|c|}{ Starter (1 to $22 \mathrm{~d}$ ) } & \multicolumn{2}{|c|}{ Grower ( 22 to $42 \mathrm{~d}$ ) } \\
\hline & $\mathrm{AFB}_{1}$ & $\mathrm{AFB}_{2}$ & $\mathrm{AFB}_{1}$ & $\mathrm{AFB}_{2}$ \\
\hline Contaminated corn & 152 & 25.3 & 232 & 38.7 \\
\hline Control & 2.3 & ND & 2.4 & ND \\
\hline $50 \%$ contaminated corn & 38.6 & 7.39 & 70.5 & 13.2 \\
\hline $100 \%$ contaminated corn & 84.8 & 15.2 & 135 & 24.8 \\
\hline $50 \%$ contaminated corn $+1 \%$ absorbent & 16.7 & 4.77 & 49.9 & 10.8 \\
\hline $100 \%$ contaminated corn $+1 \%$ absorbent & 28.1 & 7.88 & 100 & 20.1 \\
\hline
\end{tabular}

$A F B$, aflatoxins; ND, not detected.

0.05 statistical level.

\section{RESULTS}

\section{Dietary mycotoxin concentrations}

The naturally mycotoxin-contaminated corn mainly contained $\mathrm{AFB}_{1}$ and $\mathrm{AFB}_{2}$, while the rest of the mycotoxins $\left(\mathrm{AFG}_{1}, \mathrm{AFG}_{2}\right.$, DON, ZEA, T-2 toxin, OTA, $\mathrm{FB}_{1}$ ) were below the limit of detection (Table 3). Dietary $\mathrm{AFB}_{1}$ levels ranged from 16.7 to 84.8 $\mu \mathrm{g} / \mathrm{kg}$ in starter diet and from 49.9 to $135 \mu \mathrm{g} / \mathrm{kg}$ in grower diet, and the $\mathrm{AFB}_{2}$ concentrations were 4.77 to $15.2 \mu \mathrm{g} / \mathrm{kg}$ and 10.8 to $24.8 \mu \mathrm{g} / \mathrm{kg}$ for the two-phase diets, respectively.

\section{Growth performance}

During $\mathrm{d} 1$ to 21, dietary AFB-contaminated diets reduced $(p<0.05)$ BW, ADG, and ADFI, but increased F/G. Birds fed $100 \%$ AFB-contaminated diets had lower $(\mathrm{p}<0.05) \mathrm{ADFI}$, but higher $(\mathrm{p}<0.05) \mathrm{F} / \mathrm{G}$ than those fed $0 \%$ and $50 \%$ AFB-contaminated diets (Table 4). The addition of absorbent increased $(\mathrm{p}<0.05) \mathrm{BW}, \mathrm{ADG}$, and ADFI, whereas had no effect on F/G. There were interactions $(\mathrm{p}<0.05)$ in $\mathrm{BW}, \mathrm{ADG}$, and ADFI between contaminated corn and absorbent.

During d 22 to 42, ADG, ADFI, and F/G were not affected by dietary contaminated corn or absorbent. No interaction was observed between contaminated corn and absorbent.

Throughout the whole experiment, birds fed 100\% AFBcontaminated diets had lower $(\mathrm{p}<0.05)$ final BW and ADG, but higher $(\mathrm{p}<0.05) \mathrm{F} / \mathrm{G}$ compared to those fed control diets. The addition of absorbent did not affect ADG, ADFI, or F/G. There was no interaction between contaminated corn and

Table 4. Growth performance of broiler chicks fed varying contents of contaminated corn with or without absorbent

\begin{tabular}{|c|c|c|c|c|c|c|c|c|c|c|c|c|}
\hline \multirow{2}{*}{ Items } & \multicolumn{3}{|c|}{ BW (g/bird) } & \multicolumn{3}{|c|}{ ADG (g/bird } & \multicolumn{3}{|c|}{ ADFI (g/bird) } & \multicolumn{3}{|c|}{ F/G } \\
\hline & $1 \mathrm{~d}$ & $21 \mathrm{~d}$ & $42 d$ & $1-21 d$ & $22-42 d$ & $1-42 d$ & $1-21 d$ & $22-42 d$ & $1-42 d$ & $1-21 d$ & $22-42 d$ & $1-42 d$ \\
\hline \multicolumn{13}{|l|}{ Dietary treatment } \\
\hline $50 \%$ contaminated corn & 45.1 & $809^{b}$ & 2,471 & $36.8^{b}$ & 78.6 & 57.9 & $52.9^{b}$ & 163 & 105 & 1.45 & 2.08 & 1.82 \\
\hline $100 \%$ contaminated corn & 45.1 & $750^{c}$ & 2,300 & $34.1^{c}$ & 73.8 & 53.9 & $50.1^{c}$ & 160 & 102 & 1.48 & 2.16 & 1.88 \\
\hline Control+1\% absorbent & 45.1 & $822^{a b}$ & 2,457 & $37.4^{\mathrm{ab}}$ & 77.8 & 57.6 & $53.9^{\mathrm{ab}}$ & 161 & 103 & 1.45 & 2.07 & 1.80 \\
\hline $100 \%$ contaminated corn $+1 \%$ absorbent & 45.1 & $812^{b}$ & 2,385 & $36.9^{b}$ & 74.5 & 55.8 & $53.8^{\mathrm{ab}}$ & 160 & 103 & 1.47 & 2.16 & 1.86 \\
\hline SEM & & 4.31 & 17.2 & 0.22 & 0.71 & 0.40 & 0.24 & 1.36 & 0.66 & 0.01 & 0.02 & 0.01 \\
\hline \multicolumn{13}{|l|}{ Main effect mean } \\
\hline \multicolumn{13}{|l|}{ Contaminated corn (\%) } \\
\hline 0 & 45.1 & $826^{a}$ & $2,445^{\mathrm{a}}$ & $37.6^{\mathrm{a}}$ & 77.3 & $57.3^{\mathrm{a}}$ & $53.9^{\mathrm{a}}$ & 162 & 104 & $1.44^{b}$ & 2.10 & $1.82^{b}$ \\
\hline 1 & 45.1 & $818^{b}$ & 2,411 & $37.2^{b}$ & 75.7 & 56.5 & $53.9^{b}$ & 161 & 104 & 1.46 & 2.13 & 1.84 \\
\hline \multicolumn{13}{|l|}{ Source of variation, $p$-value } \\
\hline Contaminated corn & - & 0.03 & 0.05 & 0.03 & 0.36 & 0.05 & 0.04 & 0.70 & 0.42 & 0.01 & 0.29 & 0.02 \\
\hline Absorbent & - & 0.02 & 0.05 & 0.04 & 0.27 & 0.05 & 0.03 & 0.68 & 0.59 & 0.78 & 0.31 & 0.70 \\
\hline Contaminated corn $\times$ absorbent & - & 0.02 & 0.15 & 0.03 & 0.42 & 0.18 & 0.03 & 0.86 & 0.49 & 0.27 & 0.18 & 0.16 \\
\hline
\end{tabular}

Means represent 10 cages per treatment and 20 birds per cage.

$B W$, body weight; $A D G$, average daily gain; $A D F I$, average daily feed intake; $F / G$, feed-to-gain ratio; $S E M$, pooled standard error of the means.

${ }^{a-c}$ Means in a column with different superscripts are significantly different $(p<0.05)$. 
absorbent for any measures.

\section{Blood profiles}

On d 14, feeding AFB-contaminated diets did not affect serum TP, ALB, AST, ALT, AKP, or $\gamma$-GT levels (Tables 5, 6). The addition of absorbent increased $(\mathrm{p}<0.05)$ serum ALB, but reduced $(\mathrm{p}<0.05)$ serum ALT without any effect on TP, AST, or $\gamma$-GT. There was no interaction between contaminated corn and absorbent.

On d 28, the AFB-contaminated diets did not influence serum TP, ALB, AST, AKP, or $\gamma$-GT, whereas birds fed $100 \%$ AFB-contaminated diets had higher $(\mathrm{p}<0.05)$ serum ALT than those fed $0 \%$ and $50 \%$ AFB-contaminated diets. The absorbent supplementation increased $(\mathrm{p}<0.05)$ serum TP and ALB, but decreased $(\mathrm{p}<0.05)$ serum AST and AKP without any effect on ALT or $\gamma$-GT. There was no interaction between contaminated corn and absorbent except serum $\gamma$-GT $(\mathrm{p}<0.05)$

On d 42, birds fed 100\% AFB-contaminated diets had higher $(p<0.05)$ serum AKP than those fed $0 \%$ and 50\% AFBcontaminated diets. Dietary treatments did not affect serum TP, ALB, AST, ALT, or $\gamma$-GT. There was no interaction between contaminated corn and absorbent.

\section{Liver weight and total protein}

The AFB-contaminated diets and absorbent had no effect on the relative liver weight throughout the entire experiment (Table 7). Feeding AFB-contaminated diets reduced $(\mathrm{p}<0.05)$ hepatic TP on d 28 and 42 . There was no interaction between contaminated corn and absorbent.

\section{Liver gene expression}

The AFB-contaminated diets increased $(\mathrm{p}<0.05)$ the expression of IL-6, CAT, SOD, and GSH-Px, but reduced $(\mathrm{p}<0.05)$ that of GST (Table 8). The addition of absorbent increased $(p<0.05)$ the expression of IL-1 $\beta$, CAT, SOD, CYP1A1, and GST. Significant interaction of contaminated corn and absorbent was found for the expression of CAT, SOD, and GST ( $\mathrm{p}<$ 0.05).

\section{DISCUSSION}

\section{Dietary mycotoxin concentrations}

Remarkably, the contents of $\mathrm{AFB}_{1}$ and $\mathrm{AFB}_{2}$ were reduced by $60 \%$ and $40 \%$ or $68 \%$ and $51 \%$ in the starter $50 \%$ AFBcontaminated diets with adsorbent or $100 \%$ contaminated diets, respectively. In addition, the level of $\mathrm{AFB}_{1}$ and $\mathrm{AFB}_{2}$ in the grower 50\% AFB-contaminated diets and 100\% AFBcontaminated diets were decreased by $31 \%$ and $20 \%$ or $26 \%$ and $20 \%$, respectively. This indicated that the absorbent (complex activated carbon and HSCAS) successfully absorbed AFB, which was consistent with previous studies [11,12]. Moreover,

Table 5. Serum biochemical parameters of broiler chicks fed varying contents of contaminated corn with or without absorbent

\begin{tabular}{|c|c|c|c|c|c|c|c|c|c|c|c|c|}
\hline \multirow{2}{*}{ Items } & \multicolumn{3}{|c|}{$\mathrm{TP}(\mathrm{mg} / \mathrm{mL})$} & \multicolumn{3}{|c|}{ ALB $(g / L)$} & \multicolumn{3}{|c|}{ ALT (IU/L) } & \multicolumn{3}{|c|}{ AST (IU/L) } \\
\hline & $14 \mathrm{~d}$ & $28 \mathrm{~d}$ & $42 d$ & $14 \mathrm{~d}$ & $28 d$ & $42 d$ & $14 \mathrm{~d}$ & $28 d$ & $42 d$ & $14 \mathrm{~d}$ & $28 d$ & $42 d$ \\
\hline \multicolumn{13}{|l|}{ Dietary treatment } \\
\hline Control & 18.2 & 26.6 & 27.0 & 10.2 & $15.6^{\mathrm{abc}}$ & 12.9 & 4.26 & $1.28^{c}$ & 3.08 & 20.1 & 20.2 & $25.6^{\mathrm{ab}}$ \\
\hline $50 \%$ contaminated corn & 16.0 & 24.3 & 25.0 & 9.0 & $14.5^{\mathrm{bc}}$ & 12.6 & 4.02 & $2.82^{\mathrm{a}}$ & 3.61 & 21.5 & 21.2 & $22.1^{\mathrm{b}}$ \\
\hline $100 \%$ contaminated corn & 14.6 & 24.4 & 25.3 & 8.2 & $14.0^{c}$ & 12.4 & 4.40 & $2.13^{\mathrm{abc}}$ & 3.32 & 21.01 & 19.7 & $26.8^{\mathrm{a}}$ \\
\hline Control+1\% absorbent & 17.1 & 26.7 & 24.5 & 10.3 & $16.1^{\mathrm{ab}}$ & 11.9 & 3.15 & $2.51^{\mathrm{abc}}$ & 3.05 & 21.9 & 19.5 & $22.8^{b}$ \\
\hline $50 \%$ contaminated corn $+1 \%$ absorbent & 18.1 & 28.6 & 29.1 & 11.4 & $16.8^{\mathrm{a}}$ & 13.9 & 2.43 & $3.15^{\mathrm{a}}$ & 2.91 & 21.6 & 17.5 & $24.0^{a b}$ \\
\hline $100 \%$ contaminated corn $+1 \%$ absorbent & 19.0 & 25.6 & 30.4 & 11.4 & $16.2^{\mathrm{ab}}$ & 14.4 & 2.95 & $1.68^{\mathrm{bc}}$ & 3.19 & 22.2 & 17.0 & $23.2^{a b}$ \\
\hline SEM & 0.55 & 0.45 & 0.80 & 0.34 & 0.31 & 0.28 & 0.27 & 0.18 & 0.20 & 0.45 & 0.38 & 0.50 \\
\hline \multicolumn{13}{|l|}{ Main effect mean } \\
\hline \multicolumn{13}{|l|}{ Contaminated corn (\%) } \\
\hline 0 & 17.6 & 26.6 & 25.8 & 10.3 & 15.8 & 12.4 & 3.71 & $1.90^{\mathrm{b}}$ & 3.07 & 21.0 & 19.9 & 24.2 \\
\hline 50 & 17.0 & 26.5 & 27.1 & 10.2 & 15.6 & 13.2 & 3.23 & $1.90^{\mathrm{b}}$ & 3.26 & 21.6 & 19.4 & 23.1 \\
\hline 100 & 16.8 & 25.0 & 27.8 & 9.83 & 15.1 & 13.4 & 3.68 & $2.99^{\mathrm{a}}$ & 3.25 & 21.6 & 18.3 & 25.1 \\
\hline \multicolumn{13}{|l|}{ Absorbent (\%) } \\
\hline 0 & 16.3 & $25.1^{b}$ & 25.8 & $9.2^{b}$ & $14.7^{b}$ & 12.6 & $4.23^{\mathrm{a}}$ & 2.08 & 3.33 & 20.9 & $20.4^{\mathrm{a}}$ & 24.8 \\
\hline 1 & 18.1 & $27.0^{\mathrm{a}}$ & 27.9 & $11.0^{\mathrm{a}}$ & $16.4^{\mathrm{a}}$ & 13.4 & $2.85^{\mathrm{b}}$ & 2.45 & 3.05 & 21.9 & $18.0^{\mathrm{b}}$ & 23.4 \\
\hline \multicolumn{13}{|l|}{ Source of variation, $p$-value } \\
\hline Contaminated corn & 0.80 & 0.20 & 0.48 & 0.87 & 0.49 & 0.35 & 0.68 & 0.04 & 0.89 & 0.78 & 0.28 & 0.22 \\
\hline Absorbent & 0.11 & 0.04 & 0.19 & 0.02 & 0.03 & 0.19 & 0.02 & 0.16 & 0.43 & 0.30 & 0.02 & 0.18 \\
\hline Contaminated corn $\times$ absorbent & 0.18 & 0.11 & 0.15 & 0.20 & 0.33 & 0.09 & 0.88 & 0.05 & 0.72 & 0.81 & 0.25 & 0.05 \\
\hline
\end{tabular}

Means represent 10 cages per treatment and 2 birds per pen.

TP, total protein; ALB, albumin; ALT, alanine transaminase; AST, aspartate aminotransferase; SEM, pooled standard error of the means.

${ }^{a-c}$ Means in a column with different superscripts are significantly different $(p<0.05)$. 
Table 6. Serum biochemical parameters of broiler chicks fed varying contents of contaminated corn with or without absorbent

\begin{tabular}{|c|c|c|c|c|c|c|}
\hline \multirow{2}{*}{ Items } & \multicolumn{3}{|c|}{$\operatorname{AKP}(\mathrm{U} / \mathrm{dL})$} & \multicolumn{3}{|c|}{$\gamma$-GT (U/L) } \\
\hline & $14 \mathrm{~d}$ & $28 \mathrm{~d}$ & $42 \mathrm{~d}$ & $14 \mathrm{~d}$ & $28 \mathrm{~d}$ & $42 \mathrm{~d}$ \\
\hline \multicolumn{7}{|l|}{ Dietary treatment } \\
\hline Control & 368 & 380 & 93.7 & 13.2 & $31.6^{\mathrm{a}}$ & 32.8 \\
\hline $50 \%$ contaminated corn & 340 & 421 & 264 & 13.0 & $20.0^{c}$ & 34.1 \\
\hline $100 \%$ contaminated corn & 320 & 373 & 187 & 13.9 & $25.5^{\mathrm{cb}}$ & 35.1 \\
\hline Control+1\% absorbent & 214 & 317 & 119 & 14.4 & $24.3^{\mathrm{bc}}$ & 33.4 \\
\hline $50 \%$ contaminated corn $+1 \%$ absorbent & 388 & 334 & 247 & 15.2 & $28.8^{\mathrm{ab}}$ & 39.7 \\
\hline $100 \%$ contaminated corn $+1 \%$ absorbent & 219 & 175 & 112 & 15.8 & $27.9^{\mathrm{ab}}$ & 43.4 \\
\hline SEM & 27.1 & 26.9 & 23.2 & 0.53 & 0.80 & 1.48 \\
\hline \multicolumn{7}{|l|}{ Main effect mean } \\
\hline \multicolumn{7}{|l|}{ Contaminated corn (\%) } \\
\hline 0 & 291 & 348 & $106^{\mathrm{b}}$ & 13.8 & 27.9 & 33.1 \\
\hline 50 & 364 & 378 & $150^{b}$ & 14.1 & 24.4 & 36.9 \\
\hline 100 & 270 & 274 & $256^{\mathrm{a}}$ & 14.9 & 26.7 & 39.2 \\
\hline \multicolumn{7}{|l|}{ Absorbent (\%) } \\
\hline 0 & 343 & $391^{\mathrm{a}}$ & 182 & 13.4 & 25.7 & 34.0 \\
\hline 1 & 274 & $275^{b}$ & 159 & 15.1 & 27.0 & 38.8 \\
\hline \multicolumn{7}{|l|}{ Source of variation, p-value } \\
\hline Contaminated corn & 0.19 & 0.28 & 0.02 & 0.65 & 0.09 & 0.36 \\
\hline Absorbent & 0.39 & 0.21 & 0.19 & 0.80 & 0.07 & 0.22 \\
\hline Contaminated corn $\times$ absorbent & 0.34 & 0.52 & 0.76 & 0.81 & 0.04 & 0.52 \\
\hline
\end{tabular}

Means represent 10 cages per treatment and 2 birds per pen.

AKP, alkaline phosphatase; $\gamma$-GT, $\gamma$-glutamyl transferase; SEM, pooled standard error of the means.

${ }^{a-c}$ Means in a column with different superscripts are significantly different $(p<0.05)$.

Table 7. Relative liver weights and liver TP of broiler chicks fed varying contents of contaminated corn with or without absorbent

\begin{tabular}{|c|c|c|c|c|c|c|}
\hline \multirow{2}{*}{ Items } & \multicolumn{3}{|c|}{ Relative liver weight (g/kg) } & \multicolumn{3}{|c|}{ Liver TP (mg/100 mg) } \\
\hline & $14 \mathrm{~d}$ & $28 \mathrm{~d}$ & $42 \mathrm{~d}$ & $14 \mathrm{~d}$ & $28 \mathrm{~d}$ & $42 d$ \\
\hline \multicolumn{7}{|l|}{ Dietary treatment } \\
\hline Control & 26.3 & 21.8 & 26.8 & 9.91 & 8.63 & 9.51 \\
\hline $50 \%$ contaminated corn & 26.9 & 22.7 & 26.5 & 9.52 & 7.88 & 8.40 \\
\hline $100 \%$ contaminated corn & 24.7 & 23.4 & 29.7 & 9.26 & 7.60 & 7.63 \\
\hline Control+1\% absorbent & 25.6 & 21.6 & 25.1 & 9.74 & 8.82 & 8.87 \\
\hline $50 \%$ contaminated corn $+1 \%$ absorbent & 24.8 & 22.3 & 27.6 & 9.45 & 7.95 & 8.57 \\
\hline $100 \%$ contaminated corn $+1 \%$ absorbent & 26.3 & 22.9 & 26.8 & 9.66 & 8.43 & 8.28 \\
\hline SEM & 0.31 & 0.46 & 0.52 & 0.13 & 0.14 & 0.16 \\
\hline \multicolumn{7}{|l|}{ Main effect mean } \\
\hline \multicolumn{7}{|l|}{ Contaminated corn (\%) } \\
\hline 0 & 26.0 & 21.7 & 25.9 & 9.82 & $8.72^{\mathrm{a}}$ & $9.19^{a}$ \\
\hline 50 & 25.9 & 22.5 & 27.1 & 9.49 & $8.02^{b}$ & $8.48^{\mathrm{b}}$ \\
\hline 100 & 25.5 & 23.1 & 28.2 & 9.46 & $7.92^{b}$ & $7.95^{\mathrm{b}}$ \\
\hline \multicolumn{7}{|l|}{ Absorbent (\%) } \\
\hline 0 & 26.0 & 22.6 & 27.7 & 9.56 & 8.04 & 8.51 \\
\hline 1 & 25.6 & 22.3 & 26.5 & 9.62 & 8.40 & 8.57 \\
\hline \multicolumn{7}{|l|}{ Source of variation, $p$-value } \\
\hline Contaminated corn & 0.77 & 0.48 & 0.26 & 0.51 & 0.04 & 0.03 \\
\hline Absorbent & 0.49 & 0.66 & 0.23 & 0.84 & 0.34 & 0.80 \\
\hline Contaminated corn $\times$ absorbent & 0.12 & 0.89 & 0.31 & 0.65 & 0.46 & 0.13 \\
\hline
\end{tabular}

Means represent 10 cages per treatment and 2 birds per pen.

TP, total protein; SEM, pooled standard error of the means.

$a, b$ Means in a column with different superscripts are significantly different $(p<0.05)$. 
Table 8. Liver gene expression of broiler fed varying contents of contaminated corn with or without absorbent

\begin{tabular}{|c|c|c|c|c|c|c|c|c|c|}
\hline Items & IL-6 & $I L-1 \beta$ & IFN- $\gamma$ & $C A T$ & $S O D$ & GSH-Px & CYP1A1 & $E H$ & GST \\
\hline \multicolumn{10}{|l|}{ Dietary treatment } \\
\hline Control & $0.88^{c}$ & $0.78^{b}$ & 1.16 & $0.58^{d}$ & $0.80^{d}$ & $0.35^{d}$ & $1.10^{c}$ & 1.51 & $1.34^{\mathrm{a}}$ \\
\hline $100 \%$ contaminated corn & $1.00^{b}$ & $1.04^{\mathrm{ab}}$ & 1.46 & $2.37^{b}$ & $1.48^{c}$ & $2.15^{\mathrm{a}}$ & $1.48^{c}$ & 1.01 & $0.52^{c}$ \\
\hline Control+1\% absorbent & $0.71^{d}$ & $1.54^{\mathrm{a}}$ & 0.96 & $3.14^{\mathrm{a}}$ & $2.18^{\mathrm{a}}$ & $1.27^{\mathrm{b}}$ & $3.11^{\mathrm{a}}$ & 0.82 & $0.77^{b}$ \\
\hline $50 \%$ contaminated corn $+1 \%$ absorbent & $0.98^{b}$ & $1.42^{\mathrm{ab}}$ & 1.54 & $3.10^{\mathrm{a}}$ & $1.82^{b}$ & $0.61^{c}$ & $2.66^{\mathrm{ab}}$ & 0.88 & $1.34^{\mathrm{a}}$ \\
\hline \multicolumn{10}{|l|}{ Main effect mean } \\
\hline \multicolumn{10}{|l|}{ Contaminated corn (\%) } \\
\hline 0 & $0.80^{b}$ & 1.16 & 1.06 & $1.86^{b}$ & $1.49^{b}$ & $0.81^{b}$ & 2.11 & 1.17 & $1.06^{\mathrm{a}}$ \\
\hline 50 & $0.87^{b}$ & 1.16 & 1.10 & $2.47^{\mathrm{a}}$ & $1.63^{\mathrm{ab}}$ & $0.66^{c}$ & 1.78 & 0.72 & $1.03^{\mathrm{a}}$ \\
\hline 100 & $1.06^{\mathrm{a}}$ & 1.18 & 1.54 & $2.45^{\mathrm{a}}$ & $1.73^{\mathrm{a}}$ & $1.74^{\mathrm{a}}$ & 1.87 & 0.95 & $0.76^{b}$ \\
\hline \multicolumn{10}{|l|}{ Source of variation, $p$-value } \\
\hline Contaminated corn & 0.03 & 0.34 & 0.67 & 0.02 & 0.04 & 0.02 & 0.48 & 0.22 & 0.02 \\
\hline Absorbent & 0.25 & 0.02 & 0.31 & 0.01 & 0.03 & 0.46 & 0.01 & 0.43 & 0.04 \\
\hline Contaminated corn $\times$ absorbent & 0.43 & 0.66 & 0.14 & 0.04 & 0.02 & 0.05 & 0.17 & 0.30 & 0.04 \\
\hline
\end{tabular}

Means represent 10 cages per treatment and 2 birds per pen.

IL, interleukin; IFN- $\gamma$, interferon- $\gamma$; CAT, catalase; SOD, superoxide dismutase; GSH-PX, glutathione peroxidase; CYP1A1, cytochrome P450 1A1; EH, epoxide hydrolase; GST, glutathione S-transferase; SEM, pooled standard error of the means.

${ }_{a-d}$ Means in a column with different superscripts are significantly different $(p<0.05)$.

the results showed that the absorbent might be more effective in lower levels of AFB-contaminated diets due to the higher reduction degree. The higher $\mathrm{AFB}_{1}$ and $\mathrm{AFB}_{2}$ levels in grower diet might due to the longer storage time of the corn.

\section{Growth performance}

Several recent studies have demonstrated that naturally AFB-contaminated grains or purified AFB could results in aflatoxicosis, which might be due to anorexia, listlessness, impaired immune and liver function, altered intestinal morphology, and inhibition of protein synthesis and lipogenesis in broilers [3-6]. The effects of corn naturally contaminated with mycotoxins on health and performance of animals may have been greater than purified mycotoxin diets [25]. Significant interactions in $\mathrm{BW}, \mathrm{ADG}$, and $\mathrm{ADFI}$ between contaminated corn and absorbent were found in the present study, which implied that the absorbent ameliorated aflatoxicosis for broilers. Notwithstanding, in grower phase, probably because no significant growth depression was found in broilers fed diets with contaminated corn, the adsorbent did not present an improvement in growth performance. This indicated that aflatoxicosis exerts greater negative effects on growth performance in broilers in the starter phase and the absorbent could counteracted the negative effects. As expected, low naturally AFB-contaminated diets ( 16 to $85 \mu \mathrm{g} / \mathrm{kg} \mathrm{AFB}_{1}$ and 5 to 15 $\mu \mathrm{g} / \mathrm{kg} \mathrm{AFB}_{2}$ ) reduced BW, ADG, and ADFI, but increased F/G in broilers during starter phase, and the depression was more pronounced when the content reached $100 \%$ in the current study. Similarly, previous study reported that BW on $\mathrm{d} 21$ was decreased by low levels of $\mathrm{AFB}_{1}$ and $\mathrm{AFB}_{2}$ (16 to $82 \mu \mathrm{g} / \mathrm{kg}$ $\mathrm{AFB}_{1}$ and 3 to $14 \mu \mathrm{g} / \mathrm{kg} \mathrm{AFB}_{2}$ ) in broilers fed contaminated diets and the reduction increased with an increasing of naturally contaminated corn [4]. The increased F/G in broilers fed AFB-contaminated diets $(44.5 \mu \mathrm{g} / \mathrm{kg})$ was observed by previous studies [26]. Others also observed negative effects of AFB (1 to $5 \mathrm{mg} / \mathrm{kg}$ ) on growth performance in broilers [5$7,27]$. On the contrary, low purified $\mathrm{AFB}_{1}(50 \mu \mathrm{g} / \mathrm{kg})$ did not affect growth performance in broilers [3]. The inconsistency might be attributed to the fact that naturally contaminated AFB was more toxic than purified AFB and the different protein sources and levels also would alter protein utilization and animal responses to AFB $[13,28]$. In grower phase, the growth performance was not affected when exposed to AFB-contaminated diets, even though the $\mathrm{AFB}_{1}$ and $\mathrm{AFB}_{2}$ concentrations were higher than that in starter phase. The results indicated that younger broilers were more susceptible to AFB than older ones. This was supported by a meta-analysis literature which found that the effect of mycotoxins on growth was greater in young broilers [7]. Feeding 100\% AFB-contaminated diets reduced overall $\mathrm{BW}$ and $\mathrm{ADG}$, but increased $\mathrm{F} / \mathrm{G}$, which was partially consistent with previous study [4]. Furthermore, the adsorbent used in present study could effectively adsorb di- 
etary $\mathrm{AFB}_{1}$ and $\mathrm{AFB}_{2}$, and the adsorption rate was up to $68 \%$ in $100 \%$ AFB-contaminated diet. Moreover, the adsorbent supplementation improved BW, ADG, and ADFI in starter phase compared with AFB-contaminated diets and eliminated the growth reduction to control diets. The results agreed with previous studies, which also demonstrated that the activated carbon or HSCAS improved ADG and ADFI of the AFB-treated broilers $[9,12,29,30]$.

\section{Blood profiles}

When the liver was exposed to AFB, the hepatocytes were damaged and membrane permeability was enhanced, and the enzymes in liver (e.g. ALT, AST, and AKP) were released into the blood and consequently serum enzyme activity increased $[4,6]$. Feeding low levels of $\mathrm{AFB}_{1}$ and $\mathrm{AFB}_{2}$ contaminated diets had little impact on blood profiles except the serum ALT on d 28 and AKP on d 42, which were increased by dietary AFB in present study. ALT is a marker of liver injury and AKP is a signal for various liver disease states [31,32]. Therefore, the increase in serum ALT and AKP indicated that broiler livers were also damaged to some extent even when exposed to naturally low level of AFB contaminated diets for long time. In contrast, feeding high levels of AFB-contaminated diets (1.5 to $5 \mathrm{mg} / \mathrm{kg}$ ) reduced the levels of TP, ALB, and GLB, which may be due to the hepatotoxic effects of $\mathrm{AFB}_{1}$ characterized by the inhibition of protein synthesis and impairment of carbohydrate and lipid metabolism $[33,34]$. This inconsistency may be due to the different AFB origins (corn naturally contaminated or inoculated with purified mycotoxins) and dosage. Nevertheless, the addition of adsorbent was able to reduce serum ALT and AKP levels in broilers fed AFB-contaminated diets. The serum AST, another marker of liver injury, was also decreased by the adsorbent on $\mathrm{d} 28$. Similarly, $0.4 \%$ activated charcoal or $0.5 \%$ graphene oxide with chitosan adsorbents counteract the adverse effects of AFB-contaminated diets on serum ALT and $\gamma$-GT in broilers $[9,35]$. These results indicated that the adsorbent might improve cell integrity and prevent the release of liver enzymes into serum [5].

\section{Liver weight and total protein}

It was well documented that AFB and its metabolites mainly accumulate in liver and the liver becomes the key target organ [36]. In present study, we failed to observe any statistical change in the relative weight of livers in broiler fed low levels of $\mathrm{AFB}_{1}$ and $\mathrm{AFB}_{2}$, which agreed with previous studies $[3,4]$. However, others observed that diets naturally contaminated with $\mathrm{AFB}_{1}$ and $\mathrm{AFB}_{2}$ increased the relative weight of livers [37-39]. The lack of effect may be caused by the low levels of $\mathrm{AFB}_{1}$ and $\mathrm{AFB}_{2}$ in the diets of our study and the differences in $\mathrm{AFB}_{1}$ sensitivity of the bird population assayed in each experiment [3]. It is suggested that when dietary $\mathrm{AFB}_{1}$ ranged from 100 to $200 \mu \mathrm{g} / \mathrm{kg}$, the clinical, hematological-biochemical and histopathological changes may occur in broilers $[40,41]$. Besides, no significant differences were observed between broilers fed the control diet and those fed the diet containing absorbent alone, indicating that the adsorbent was inert and nontoxic in agreement with previous findings [3]. In the current study, with the increase of AFB-contaminated corn, hepatic TP levels decreased on d 28 and 42, and the reduction increased with time. The results were consistent with previous studies on broilers [34,39], illustrating that chronic aflatoxicosis would impair protein synthesis in liver and the impairment increased with time and AFB concentration.

\section{Liver gene expression}

Although the relative weight of livers did not change, the hepatic TP levels were decreased by the AFB-contaminated diets. $\mathrm{AFB}_{1}$ was biotransformed into various metabolites, especially the toxic $\mathrm{AFB}_{1}$-exo-8,9-epoxide (AFBO), which occurred in the liver and the intestinal tract [42]. AFBO is a potent inhibitor of protein synthesis in poultry through its interaction with DNA and RNA [43]. However, literature on AFB in this regard is still scarce. A recent study evaluated the effects of AFB-contaminated diets on mRNA expression of jejunal peptide and amino acid transporters in broilers and found that a higher mRNA production is needed to increase translation process to restore possible $\mathrm{AFB}_{1}$ or $\mathrm{AFBO}$ impaired protein activities [34]. Therefore, we supposed that liver gene expression may also be modulated by the AFB-contaminated diets and hence examined the effects of AFB-contaminated diets on liver gene expression involved in inflammatory response (IL-6, IL-1 $\beta, I F N-\gamma)$, antioxidant function (CAT, SOD, GST- $\alpha)$ and biotransformation of AFB (EH, GSH-Px, CYP1A1) in response to AFB and absorbent. The cytokines (IL-6 and IL$1 \beta$ secreted by activated macrophages and IFN $-\gamma$ originated from $T$ helper cells, natural killer cells, and macrophages) are released to induce inflammatory reactions and mediate the immune responses when exposed to infections in poultry [44]. The AFB-contaminated diets upregulated the expression of IL-6, which was similar with the results of previous studies in broilers fed 1 to $2 \mathrm{mg} / \mathrm{kg} \mathrm{AFB}{ }_{1}[5,16]$. This indicated that the $\mathrm{AFB}_{1}$-contaminated diets led to hepatic inflammatory response. In addition, the reverse response to absorbent was found for IL- $1 \beta$ in our study. Moreover, the HSCAS can partially ameliorate the inflammation by reducing IL- 6 expression in liver induced by $\mathrm{AFB}_{1}$ for broilers [5]. The CAT, SOD, and GSH-Px as the key enzymes of antioxidant system can scavenge free radicals generated from oxidant stress, reduce oxidative damage and maintain cell structure. The expression of CAT, SOD, and GSH-Px was upregulated by the AFB-contaminated diets and the absorbent supplementation also upregulated the expression of CAT, SOD, and GSH-Px. Similarly, previous study also observed that the SOD and GSH-Px expression 
in broilers was upregulated by 0.5 to $1 \mathrm{mg} / \mathrm{kg}$ purified $\mathrm{AFB}_{1}$, but they were not influenced by the absorbent (HSCAS) [5]. The results were not always consistent. No significant change in GSH-Px expression in response to $\mathrm{AFB}_{1}$ was observed [16]. AFBO can be detoxified primarily by GST enzymes by forming glutathione conjugates, or to a lesser extent by $\mathrm{EH}$ through a conversion to $\mathrm{AFB}_{1}$-dihydrodiol [45]. In our study, the AFBcontaminated diets downregulated GST expression, while the addition of absorbent increased the expression GST, which indicated that the absorbent may be effective in detoxifying. On the contrary, feeding $2 \mathrm{mg} / \mathrm{kg} \mathrm{AFB}{ }_{1}$-contaminated diets increased the expression of $\mathrm{EH}$ and GST, which indicated an increase in AFBO accumulation [5]. CYP1A1 is a member of the CYP450 enzyme family and is one of the enzymes that is responsible for activating $\mathrm{AFB}_{1}$. The addition of absorbent increased CYP1A1 expression, which agreed with previous study [5]. In contrast, feeding $1 \mathrm{mg} / \mathrm{kg} \mathrm{AFB}{ }_{1}$-contamianted diets upregulated CYP1A1 expression in broilers [16]. The inconsistency may be due to different AFB levels and origins. The current results also demonstrated that the $\mathrm{AFB}_{1}$-contaminated diets damaged the hepatic tissues at the molecular level.

\section{CONCLUSION}

The naturally $\mathrm{AFB}_{1}$ and $\mathrm{AFB}_{2}$ contaminated diets depressed growth performance, especially in the starter phase and negatively affected blood profiles, while increased the expression of IL-6, CAT, SOD, and GSH-Px in liver of in broilers. The adsorbent (complex activated carbon and HSCAS) could effectively adsorb dietary $\mathrm{AFB}_{1}$ and $\mathrm{AFB}_{2}$ and alleviated aflatoxicosis by improving $\mathrm{ADG}$ and $\mathrm{ADFI}$ in the starter phase and partially restoring the negatively influenced blood profiles and hepatic gene expression in young broilers.

\section{CONFLICT OF INTEREST}

We certify that there is no conflict of interest with any financial organization regarding the material discussed in the manuscript.

\section{ACKNOWLEDGMENTS}

This study was supported by the National Key Research and Development Program of China (2016YFD0500505) and Sichuan Science and Technology Program (2018HH0083).

\section{REFERENCES}

1. Gourama H, Bullerman LB. Aspergillus flavus and Aspergillus parasiticus: Aflatoxigenic fungi of concern in foods and feeds: a review. J Food Protect 1995;58:1395-404. https://doi.org/10.

\section{5/0362-028X-58.12.1395}

2. Nilipour A. Mycotoxins, an insidious global concern. World Poult 2002;2:18-20.

3. Magnoli AP, Monge MP, Miazzo RD, et al. Effect of low levels of aflatoxin $B_{1}$ on performance, biochemical parameters, and aflatoxin $B_{1}$ in broiler liver tissues in the presence of monensin and sodium bentonite. Poult Sci 2011;90:48-58. https://doi. org/10.3382/ps.2010-00971

4. Yang J, Bai F, Zhang $\mathrm{K}$, et al. Effects of feeding corn naturally contaminated with aflatoxin $\mathrm{B}_{1}$ and $\mathrm{B}_{2}$ on hepatic functions of broilers. Poult Sci 2012;91:2792-801. https://doi.org/10.3382/ ps.2012-02544

5. Chen X, Horn N, Applegate TJ. Efficiency of hydrated sodium calcium aluminosilicate to ameliorate the adverse effects of graded levels of aflatoxin B1 in broiler chicks. Poult Sci 2014; 93:2037-47. https://doi.org/10.3382/ps.2014-03984

6. Chen X, Zhang Q, Applegate T. Impact of dietary branched chain amino acids concentration on broiler chicks during aflatoxicosis. Poult Sci 2016;95:1281-9. https:/doi.org/10.382/ ps/pew061

7. Andretta I, Kipper M, Lehnen C, Hauschild L, Vale MM, Lovatto PA. Meta-analytical study of productive and nutritional interactions of mycotoxins in broilers. Poult Sci 2011;90:193440. https://doi.org/10.3382/ps.2011-01470

8. Shi D, Zhou JC, Zhao LH, et al. Alleviation of mycotoxin biodegradation agent on zearalenone and deoxynivalenol toxicosis in immature gilts. J Anim Sci Biotechnol 2018;9:42. https:// doi.org/10.1186/s40104-018-0255-Z

9. Saminathan M, Selamat J, Abbasi Pirouz A, Abdullah N, Zulkifli I. Effects of nano-composite adsorbents on the growth performance, serum biochemistry, and organ weights of broilers fed with aflatoxin-contaminated feed. Toxins 2018;10:345. https:// doi.org/10.3390/toxins10090345

10.Li Y, Tian G, Dong G, et al. Research progress on the raw and modified hydrated sodium calcium aluminosilicates as adsorbents for mycotoxins: a review. Appl Clay Sci 2018;163:299311. https://doi.org/10.1016/j.clay.2018.07.032

11.Pappas A, Tsiplakou E, Georgiadou M, et al. Bentonite binders in the presence of mycotoxins: results of in vitro preliminary tests and an in vivo broiler trial. Appl Clay Sci 2014;99:48-53. https://doi.org/10.1016/j.clay.2014.06.009

12. Khadem A, Sharifi S, Barati M, et al. Evaluation of the effectiveness of yeast, zeolite and active charcoal as aflatoxin absorbents in broiler diets. Global Vet 2012;4:426-32.

13. Whitlow L, Hagler W, Diaz D. Mycotoxins in feeds. Feedstuffs 2002;74:74-84.

14. Bai S, Wang L, Luo Y, et al. Effects of corn naturally contaminated with aflatoxins on performance, calcium and phosphorus metabolism, and bone mineralization of broiler chicks. J Poult Sci 2014;51:157-64. https://doi.org/10.2141/jpsa.0130053

15. Miazzo R, Rosa C, de Queiroz CE, et al. Efficacy of synthetic zeolite to reduce the toxicity of aflatoxin in broiler chicks. Poult 
Sci 2000;79:1-6. https://doi.org/10.1093/ps/79.1.1

16. Yarru LP, Settivari RS, Gowda NKS, Antoniou E, Ledoux DR, Rottinghaus GE. Effects of turmeric (Curcuma longa) on the expression of hepatic genes associated with biotransformation, antioxidant, and immune systems in broiler chicks fed aflatoxin. Poult Sci 2009;88:2620-7. https://doi.org/10.3382/ps.200900204

17. National Research Council. Nutrient requirements of poultry. 9th revised. Washington, DC, USA: National Academy Press; 1994.

18. AOAC. Official methods of analysis of AOAC International. 17th ed. Gaithersburg, MD, USA: AOAC International; 1995.

19.Liu JB, Xue PC, Cao SC, Liu J, Chen L, Zhang HF. Effects of dietary phosphorus concentration and body weight on postileal phosphorus digestion in pigs. Anim Feed Sci Technol 2018; 242:86-94. https://doi.org/10.1016/j.anifeedsci.2018.06.003

20.Liu JB, Yan HL, Cao SC, Liu J, Li ZX, Zhang HF. The response of performance in grower and finisher pigs to diets formulated to different tryptophan to lysine ratios. Livest Sci 2019;222: 25-30. https://doi.org/10.1016/j.livsci.2019.01.016

21.Liu JB, Yan HL, Zhang Y, et al. Effects of dietary energy and protein content and lipid source on growth performance and carcass traits in Pekin ducks. Poult Sci 2019;98:4829-37. https:// doi.org/10.3382/ps/pez217

22. Yan HL, Zhang L, Guo ZD, Zhang H, Liu J. Production phase affects the bioaerosol microbial composition and functional potential in swine confinement buildings. Animals 2019;9:90. https://doi.org/10.3390/ani9030090

23. Yan HL, Cao SC, Li Y, Zhang H, Liu J. Reduced meal frequency alleviates high-fat diet-induced lipid accumulation and inflammation in adipose tissue of pigs under the circumstance of fixed feed allowance. Eur J Nutr 2019 Feb 12 [Epub]. https:// doi.org/10.1007/s00394-019-01928-3

24.Livak KJ, Schmittgen TD. Analysis of relative gene expression data using real-time quantitative PCR and the $2^{-\Delta \Delta \mathrm{Ct}}$ method. Methods 2001;25:402-8. https://doi.org/10.1006/meth.2001. 1262

25. He J, Zhang KY, Chen DW, Ding XM, Feng GD, Ao X. Effects of maize naturally contaminated with aflatoxin $\mathrm{B} 1$ on growth performance, blood profiles and hepatic histopathology in ducks. Livest Sci 2013;152:192-9. https://doi.org/10.1016/j. livsci.2012.12.019

26. Resanovic R, Sinovec Z. Effects of limited feeding of aflatoxin B1 contaminated feed on the performance of broilers. Mycotoxin Res 2006;22:183-8. https://doi.org/10.1007/BF02959274

27.Zhao J, Shirley RB, Dibner JD, et al. Comparison of hydrated sodium calcium aluminosilicate and yeast cell wall on counteracting aflatoxicosis in broiler chicks. Poult Sci 2010;89:214756. https://doi.org/10.3382/ps.2009-00608

28. Chen X, Naehrer K, Applegate TJ. Interactive effects of dietary protein concentration and aflatoxin $\mathrm{B} 1$ on performance, nutrient digestibility, and gut health in broiler chicks. Poult Sci
2016;95:1312-25. https://doi.org/10.3382/ps/pew022

29. Shannon TA, Ledoux DR, Rottinghaus GE, Shaw DP, Dakovic A, Markovic M. The efficacy of raw and concentrated bentonite clay in reducing the toxic effects of aflatoxin in broiler chicks. Poult Sci 2017;96:1651-8. https://doi.org/10.3382/ps/ pew408

30. Wang XH, Li W, Wang XH, et al. Water-soluble substances of wheat: a potential preventer of aflatoxin B1-induced liver damage in broilers. Poult Sci 2019;98:136-49. https://doi.org/ $10.3382 /$ ps/pey358

31. Srivastava AR, Kumar S, Agarwal GG, Ranjan P. Blunt abdominal injury: serum ALT-A marker of liver injury and a guide to assessment of its severity. Injury 2007;38:1069-74. https:// doi.org/10.1016/j.injury.2007.04.019

32. Kim WR, Flamm SL, Di Bisceglie AM, et al. Serum activity of alanine aminotransferase (ALT) as an indicator of health and disease. Hepatology 2008;47:1363-70. https://doi.org/10. 1002/hep.22109

33. Rosa CAR, Miazzo R, Magnoli C, et al. Evaluation of the efficacy of bentonite from the south of Argentina to ameliorate the toxic effects of aflatoxin in broilers. Poult Sci 2001;80:139-44. https://doi.org/10.1093/ps/80.2.139

34. Chen X, Naehrer K, Applegate TJ. Interactive effects of dietary protein concentration and aflatoxin $\mathrm{B} 1$ on performance, nutrient digestibility, and gut health in broiler chicks. Poult Sci 2016;95:1312-25. https://doi.org/10.3382/ps/pew022

35. Jayasri A, Srikanth NR. Combined effect of aflatoxin and ochratoxin on liver enzymes of broilers and amelioration using adsorbents. J Livest Sci 2016;7:26-9.

36. Quezada T, Cuellar H, Jaramillo-Juarez F, et al. Effects of aflatoxin $\mathrm{B} 1$ on the liver and kidney of broiler chickens during development. Comp Biochem Phys C. 2000;125:265-72.

37. Kumar R, Balachandran C. Histopathological changes in broiler chickens fed aflatoxin and cyclopiazonic acid. Vet Arhiv 2009; 79:31-40.

38. Safameher, A. Effects of clinoptilolite on performance, biochemical parameters and hepatic lesions in broiler chickens during aflatoxosis. J Anim Vet Adv 2008;7:381-8.

39. Sakhare PS, Harne SD, Kalorey DR, Warke SR, Bhandarkar AG, Kurkur NV. Effect of Toxiroak ${ }^{\circledR}$ polyherbal feed supplement during induced aflatoxicosis, ochratoxicosis and combined mycotoxicoses in broilers. Vet Arhiv 2007;77:129-46.

40.Shi Y, Xu Z, Sun Y, Wang C, Feng J. Effects of two different types of motmorillonite on growth performance and serum profiles of broiler chicks during aflatoxicosis. Turk J Vet Anim Sci 2009;33:15-20.

41. Oğuz H, Kececi T, Birdane YO, Onder F, Kurtoğlu V. Effect of clinoptilolite on serum biochemical and haematological characters of broiler chickens during aflatoxicosis. Res Vet Sci 2000;69:89-93. https://doi.org/10.1053/rvsc.2000.0395

42. Sergent T, Ribonnet 1, Kolosova A, et al. Molecular and cellular effects of food contaminants and secondary plant components 
and their plausible interactions at the intestinal level. Food Chem Toxicol 2008;46:813-41. https://doi.org/10.1016/j.fct. 2007.12.006

43. Grenier B, Applegate TJ. Modulation of intestinal functions following mycotoxin ingestion: Meta-analysis of published experiments in animals. Toxins 2013;5:396-430. https://doi. org/10.3390/toxins5020396

44. Klasing KC. Avian leukocytic cytokines. Poult Sci 1994;73: 1035-43. https://doi.org/10.3382/ps.0731035

45.Eaton DL, Gallagher EP. Mechanisms of aflatoxin carcinogenesis. Annu Rev Pharmacol Toxicol 1994;34:135-72. https:// doi.org/10.1146/annurev.pa.34.040194.001031 